\title{
Nocturnal enuresis in children - Treatment with acupuncture
}

\author{
Jihe Zhu ${ }^{1}$, Blagica Arsovska ${ }^{2}$, Kristina Kozovska ${ }^{3}$ \\ ${ }^{1,3}$ Faculty of Medical Sciences, University Goce Delcev - Shtip, Republic of Macedonia; \\ ${ }^{2}$ Institute of Biology, Faculty of Natural Sciences and Mathematics - Skopje, Republic of Macedonia;
}

\begin{abstract}
Nocturnal enuresis or bedwetting is a widespread and distressing condition that can have a deep impact on the children's behavior, emotional wellbeing and social life. Acupuncture treatment based on the Traditional Chinese Medicine theory has been commonly used to treat nocturnal enuresis. The main purpose of this work is to show a multiple cases of nocturnal enuresis in children, the effects and results from the acupuncture treatment. 13 children were treated, of which 8 male and 5 female. The children were on age from 6 to 22. Treatments were done on same acupoints, indoor on a room temperature, with duration of 35-40 minutes. The treatments ware effective in all the children. They stopped bedwetting after on average 8 treatments. The male patients needed 8 treatments and the female 7 on average to achieve the results. There were more older female patients than male. Older children have done more treatments, as they were all a deep sleepers. Acupuncture as a treatment for nocturnal enuresis in children gives satisfying results and improves the condition significantly.
\end{abstract}

Key words: acupuncture, children, nocturnal enuresis, treatment

\section{Introduction}

Nocturnal enuresis is a worldwide health problem, frequently encountered in childhood. It is defined as an involuntary voiding of urine during sleep with a frequency of at least twice a week in children, in the absence of congenital or acquired defects of the central nervous system.[1] Nocturnal enuresis affects $5 \%$ to $10 \%$ of the younger school-age children.[2] The frequency and severity of wetting episodes progressively increases with age, and children over 10 years of age have significantly more day time urinary symptoms and incontinence.[3]

The enuresis appears as monosymptomatic, with no daytime urinary symptoms and nonmonosymptomatic which is accompanied by day time urinary symptoms. [4] Also, enuresis can be primary and secondary. Primary enuresis occurs from birth, if the child has never been dry. The secondary enuresis developed after a period of at least 6 months of dryness. There are some general predisposing factors for nocturnal enuresis: genetic predisposition, stress, constipation, changes in routine - such as moving in a new house or a new baby in the family, a physical or learning disability and etc.[5]

Dr J.C. Darling in his article for nocturnal enuresis has written about the three systems approach by Butler and Holland. The three suggested systems are:

1. Adequate functional bladder capacity - Due to detrusor muscle instability, which can be affected by anxiety, chronic stress, inadequate intake of fluids or irregular patterns of urination through the day. It results with contractions when the bladder is only partly full with reduced capacity and day time symptoms of frequency, urgent, and sometimes daytime wetting.

2. Arginine vasopressin - the anti-diuretic hormone which is produced in the hypothalamus and released by the posterior pituitary, can concentrate the urine and reduce its volume. Some children with nocturnal enuresis lack this normal nocturnal rise in arginine vasopressin which results with excessive quantities of dilute urine, thus exceeding bladder capacity.

3. Ability to wake at full bladder sensations - Children with nocturnal enuresis appear to be deep sleepers and they are unable to wake at full bladder sensations. [6]

In the Traditional Chinese Medicine, nocturnal enuresis is seen as a disorder of the water fluids. The three main viscera which control water fluids in the body are the three main viscera involved in enuresis - the lungs, spleen, and kidneys. The spleen is the root where the phlegm engenders and the lungs are the place where phlegm is accumulated. The spleen reigns over the movement and transformation of the water fluids, sending the clear part of water fluids taken in by the stomach up to the lungs to distribute around the body. The kidneys rule the water fluids of the entire body and control the transformation of bladder's Qi, the opening and closing of the urethra. If any of these viscera's function is suppressed or become vacuous and insufficient, their control of water fluids may be incapacitated and enuresis may occur. The abnormal deep sleeping of the children with enuresis is combined of spleen and kidney vacuity complicated by phlegm and dampness. [7] 


\section{Material and method}

In this article is shown a multiple cases of a group of children from the orphanage with nocturnal enuresis. 13 children were treated, of which 8 male and 5 female. The children were on age from 6 to 22 . All the children have done certain number of treatments on the same acupoints, indoor on a room temperature, with duration of the treatment of 35-40 minutes. The treatments were done in a clinic for Traditional Chinese Medicine and acupuncture in Skopje, Macedonia, by a doctor specialist in acupuncture. During the treatments are used fine, sterile acupuncture needles size $0.25 \times 25 \mathrm{~mm}$ produced by Wuijuiang City Medical \& Health Material Co., LTD. Acupoints that were treated are: DU20 (BaiHui), SiShenCong ( $3 \mathrm{~cm}$ from the point BaiHui), GB20 (FengChi), Du16 (FengFu), RN6 (QiHai), BL23 (ShenShu), BL28 (PangGuangShu).

\section{Results}

All the children that were treaded have nocturnal enuresis and were a deep sleepers. Because the children are from the orphanage there are no detailed information about since when they wet, how many times a night, are there other symptoms like polyuria. Therefore we can't conclude whether it is primary or secondary enuresis or monosymptomatic or nonmonosymptomatic. Still, all the children have positive effect from the acupuncture treatment with certain, individual number of treatments.

According to the gender of the children, there were more male patients than female i.e. 8 male - $62 \%$ and 5 female $-38 \%$. Nocturnal enuresis occurs three times more often in boys than in girls. [8] The patients were on age from 6 to 22, with average age 11. The male patients were on age from 6 to 19 and the female from 7 to 22 . There were more older female patients than male. (Fig.1)

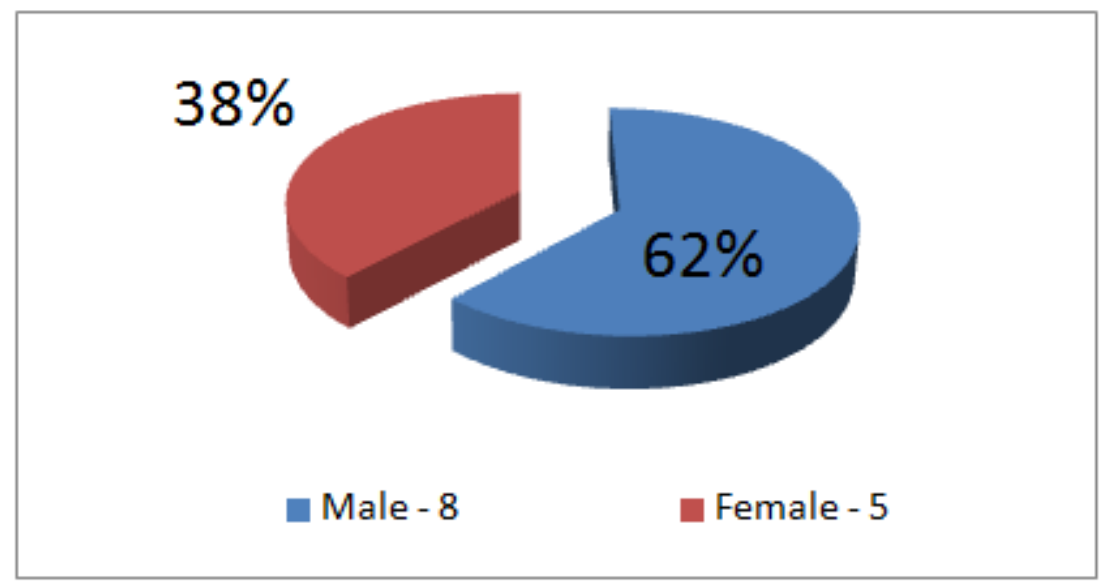

Fig1. Gender of the children

According to the different age groups and the number of children in each group, there were no children on the age of 5. There were two children in each of the groups of 6,7 and 8 years. In the groups of 9 and 10 years there was one child in each and there were five children in the group of 10 years and more. According to the treatments, older children needed more number of treatments. The male patients needed 8 therapies on average and the female 7. (Table 1)

Table1. Number of children in different age group

\begin{tabular}{|l|l|}
\hline Table 1. Number of children in different age group \\
\hline Age & Children \\
\hline 5 & 0 \\
\hline 6 & 2 \\
\hline 7 & 2 \\
\hline 8 & 2 \\
\hline 9 & 1 \\
\hline 10 & 1 \\
\hline$>10$ & 5 \\
\hline
\end{tabular}


According to the number of the treatments done, three children have done less than 6 treatments, three children have done 6 treatments, two have done 7 treatments, only one child 8 treatments and two children have done 9,10 or more treatments in each group. (Table 2)

Table2. Number of treatments

\begin{tabular}{|l|l|}
\hline Table 2. Number of treatments \\
\hline Less than 6 treatments & 3 children \\
\hline 6 treatments & 3 children \\
\hline 7 treatments & 2 children \\
\hline 8 treatments & 1 child \\
\hline 9 treatments & 2 children \\
\hline 10 and more treatments & 2 children \\
\hline
\end{tabular}

Acupuncture as a treatment for nocturnal enuresis in children is used very often and gives satisfying results. The treatment helps to restore the balance of the energy, harmonize the internal state of the body and clear the symptoms of the nocturnal enuresis.

\subsection{Cases}

Male, 11 years, wetting every night. Five treatments made in a period of three weeks. The first treatment was made on 19.05.2008. After the third treatment he reported that he was wetting on every third night. After the sixth treatment he stopped bedwetting.

Female, 7 years, wetting every night. Five treatments made in a period of three months. The first treatment was made on 15.10.2015. After the $2^{\text {nd }}$ treatment made on 29.11.2015 she reported she haven't wet three nights. After the $3^{\text {rd }}$ treatment made on 20.12.2015 she reported she haven't wet seven nights. After the $4^{\text {th }}$ treatment made on 10.01.2016 she reported she wet only three nights. And after the last treatment made on 24.01.2016 she stopped bedwetting.

Female, 22 years, wetting every night. Fourteen treatments made in a period of three months. The first treatment was made on 19.09.2009 and the last one on 27.11.2009 when she stopped bedwetting.

Male, 19 years, wetting every night. Nine treatments made, every day for nine days. The first treatment was made on 21.11.2006 and last one on 29.11.2006 when he stopped bedwetting.

\section{Conclusion}

Acupuncture as a treatment for nocturnal enuresis in children gives satisfying results and improves the condition significantly.

\section{References}

[1]. NICE clinic guideline 111; Nocturnal enuresis: the management of bedwetting in children and young people; Issue date: Oct 2010

[2]. Zheng-tao Lv, Song W, Wu J et al; Efficacy of Acupuncture in Children with Nocturnal Enuresis: A Systematic Review and MetaAnalysis of Randomized Controlled Trials; Evidence-Based Complementary and Alternative Medicine; Volume 2015; ID 320701; 2015 http://dx.doi.org/10.1155/2015/320701

[3]. Naseri M, Hiradfar M; Monosymptomatic and Non-monosymptomatic Nocturnal Enuresis: A Clinical Evaluation; Arch Iran Med.; 15(11):702-706.; 2012

[4]. F.Saettini, Marra C, Rota M et al; Acupuncture for the treatment of pediatric nocturnal enuresis: A systematic review and a metaanalysis of randomized and non-randomized studies; Eur. J. Integr. Med. 2015, http://dx.doi.org/10.1016/j.eujim.2015.08.002

[5]. Dobson P, Weaver A; Nocturnal enuresis: systems for assessment and treatment; Nurs Times. 21-27;102(12):49-52.; Mar 2006

[6]. Darling CJ; Management of nocturnal enuresis; Trends in Urology, Gynaecology \& Sexual Health; Volume 15, Issue 3; 8 June 2010

[7]. Helmer R; Treating Pediatric Bed-wetting with Acupuncture \& Chinese Medicine; Blue Poppy Press; ISBN 978-1-891845-33-0; 2006

[8]. Thiedke CC; Nocturnal enuresis; Am Fam Physician; 67(7):1499-1506; Apr 12003 